\title{
Towards controlling the dissociation probability by light-induced conical intersections
}

\author{
András Csehi, ${ }^{1}$ Gábor J. Halász, ${ }^{2}$ Lorenz S. Cederbaum, ${ }^{3}$ and Ágnes Vibók ${ }^{1,4}$ \\ ${ }^{1}$ Department of Theoretical Physics, University of Debrecen, \\ H-4010 Debrecen, PO Box 5, Hungary \\ ${ }^{2}$ Department of Information Technology, \\ University of Debrecen, H-4010 Debrecen, PO Box 12, Hungary \\ ${ }^{3}$ Theoretische Chemie, Physikalish-Chemisches Institut, \\ Universität Heidelberg, H-69120 Heidelberg, Germany \\ ${ }^{4}$ ELI-ALPS, ELI-HU Non-Profit Ltd, \\ H-6720 Szeged, Dugonics tér 13, Hungary
}

\begin{abstract}
Light-induced conical intersections (LICIs) can be formed both by standing or by running laser waves. The position of a LICI is determined by the laser frequency while the laser intensity controls the strength of the nonadiabatic coupling. Recently, it was shown within the LICI framework that linearly chirped laser pulses have an impact on the dissociation dynamics of the $D_{2}^{+}$molecule (J. Chem. Phys. 143, 014305, (2015); ibid 144, 074309, (2016)). In this work we exploit this finding and perform calculations using chirped laser pulses in which the time dependence of the laser frequency is designed so as to force the LICI to move together with the field-free vibrational wave packet as much as possible. Since nonadiabaticity is strongest in the vicinity of the conical intersection, this is the first step towards controlling the dissociation process via the LICI. Our showcase example is again the $D_{2}^{+}$molecular ion. To demonstrate the impact of the LICIs on the dynamical properties of diatomics, the total dissociation probabilities and the population of the different vibrational levels after the dissociation process are studied and discussed.
\end{abstract}




\section{INTRODUCTION}

Conical intersections (CIs) provide a particularly striking and important cause for nonadiabatic dynamics in polyatomic molecules not available in diatomics. At a CI the nonadiabatic couplings become singular and this gives rise to dramatic nonadiabatic effects widely studied in the literature [1, 2]. Clearly, the position of a CI appearing in nature and the strength of the nonadiabatic effects are inherent properties of the electronic states of a molecule and are hard to manipulate. For brevity, we rename the naturally occurring CIs "natural CIs". While exposed to resonant laser light, a new feature emerges. This feature is a CI induced by the light which cannot be avoided even in the case of diatomic molecules [3, 4]. The angle $\theta$ between the laser polarization and the molecular axis now becomes the missing dynamical variable that together with the stretching coordinate constitute the space in which the induced CI can live. The phenomenon is rather general and not restricted to propagating waves. Light-induced CIs (LICI) automatically emerge also in standing waves which form optical lattices widely used in cold-atom physics [3]. Actually, this is the case where they have been first predicted. In optical lattices not only the electronic, the stretching and rotational motion couple strongly via the induced CI, but also the translational motion enters the scene and becomes a dynamical variable. This gives cooling mechanisms new aspects. It has been demonstrated that the LICI in diatomics gives rise to a variety of nonadiabatic phenomena. Of course, they exhibit a topological phase and provide singular nonadiabatic couplings like the natural CIs do [5, 6]. Already in traveling waves of rather weak intensity the LICI has a substantial impact on the spectrum of the molecule [4]. Moreover, even in short laser pulses one finds remarkable consequences of the LICI. The degree of alignment of a diatomic molecule as a function of time differs substantially from the rigid rotor predictions [7]. A wealth of nuclear-wave-packet quantum interferences show up in intense laser dissociation due to the nonadiabatic involvement of the rotation [8, 9]. A robust effect in the angular distribution of the photofragments has been found that serves as a direct signature of the LICI and should be amenable to observations [10]. If the resonant laser excitation is to an electronically metastable state which decays by emitting an electron, the LICI is in the continuum and changes its topology like all natural CIs which are embedded in the continuum. It takes on the appearance of doubly-intersecting com- 
plex surfaces (DICES) which also exhibit singular nonadiabatic couplings [11]. Examples where light-induced DICES appear are resonant Auger decay in diatomics and interatomic Coulombic decay by two-photon transitions in intense pulses. LICIs are ubiquitous in polyatomic molecules both fixed in space or freely rotating. Because of the presence of several vibrational degrees of freedom, LICIs exist also without rotations and become multidimensional in nuclear coordinate space [12]. This opens the door for manipulating and controlling nonadiabatic effects by light [12 [17].

Currently, we are investigating the impact of linearly chirped laser pulses on the dissociation of diatomic molecules $\left(\mathrm{D}_{2}^{+}\right)$[18, 19]. As the frequency of the pulse changes in time, the position of the LICI changes analogously. It was found that using laser pulses which are long enough $\left(T_{p} \geq 10 \mathrm{fs}\right)$ the amplitude of the periodic change in the total dissociation probability is significantly squeezed by the chirped pulse compared to the transform limited situation [18]. By chirping the frequency the effective pulse duration is increased and the effective intensity is decreased by a same factor resulting in the suppression of the dissociation yields [19]. Several works in the literature demonstrate the controllability of different dynamical properties of molecular systems by using chirped laser pulses [20 43].

To be consistent with our former chirp type investigations, the showcase example will again be the $\mathrm{D}_{2}^{+}$molecule. In the present work we plan to go further and perform calculations using chirped laser pulses in which the time dependence of the laser frequency is designed so as to constrain for the motion of the LICI's position to follow, as much as possible, the time evolution of the nuclear wave packet. To achieve this goal, the time dependence of the laser frequency is approximated by the energy difference between the potential energy curves of the participating electronic states of the $\mathrm{D}_{2}^{+}$ion. We note, that the ideal case would be to let the LICI follow the true wave packet and not the field-free one. This can be done by a self-consistent procedure (optimal control theory). But, since this is the first study of its kind, we would like to first see whether there are gains to be expected from the LICI following the wave packet, and this is the purpose the present investigation.

The photodissociation probabilities and the population of the different vibrational levels after the dynamics are studied. Two dimensional (2D) simulations are applied to the problem in which the rotational angle is taken into account as a dynamic variable and, therefore, the LICI is explicitly included. 


\section{THE HAMILTONIAN}

Figure 1] shows the potential energy curves for $D_{2}^{+}$in Floquet representation [44]. $V_{1}(R)=$ $1 s \sigma_{g}, V_{2}(R)=2 p \sigma_{u}$ and $\left(V_{2}(R)-\hbar \omega_{L}\right)$ denote the energies of the electronic ground, the first excited and the dressed eigenstates, which are considered in the calculations. Assuming the scenario where the neutral $D_{2}$ molecule is ionized at $t=0$, its vibrational ground state is transferred vertically to the potential energy curve of the ground electronic state $\left(V_{1}=1 s \sigma_{g}\right)$ of the ion. This defines the initial wave packet for the nuclear dynamics, which can be considered as the Franck-Condon (FC) distribution of all the vibrational states of the ion $D_{2}^{+}$. Exciting the $1 s \sigma_{g}$ ground electronic state of the $D_{2}^{+}$molecule by a resonant laser pulse to the repulsive $2 p \sigma_{u}$ state (see also Fig. 1) these two electronic states are radiatively coupled by the electric field. An electronic transition occurs due to nonvanishing transition dipole moment and light-induced states are formed. In the space of the $V_{1}(R)$ and $V_{2}(R)$ electronic states the following time-dependent Hamiltonian can be written for the rovibronic nuclear dynamics of the $D_{2}^{+}$:

$$
\begin{gathered}
H=\left(\begin{array}{cc}
-\frac{1}{2 \mu} \frac{\partial^{2}}{\partial R^{2}}+\frac{L_{\theta \varphi}^{2}}{2 \mu R^{2}} & 0 \\
0 & -\frac{1}{2 \mu} \frac{\partial^{2}}{\partial R^{2}}+\frac{L_{\theta \varphi}^{2}}{2 \mu R^{2}}
\end{array}\right)+ \\
\left(\begin{array}{cc}
V_{1}(R) & -E(t) d(R) \cos \theta \\
-E(t) d(R) \cos \theta & V_{2}(R)
\end{array}\right) .
\end{gathered}
$$

The off-diagonal elements of eq. (2.1) represent the radiative couplings, where the electric field $E(t)$ characterizes the laser field. $d(R)\left(=-\left\langle\psi_{1}^{e}\left|\sum_{j} r_{j}\right| \psi_{2}^{e}\right\rangle\right)$ is the transition dipole matrix element and $\theta$ is the angle between the polarization direction of the light and the direction of the molecular axes. Here, $\mathrm{R}$ and $(\theta, \varphi)$ are the molecular vibrational and rotational coordinates, respectively, $\mu$ is the reduced mass, and $L_{\theta \varphi}$ is the angular momentum operator of the nuclei ( $e=m_{e}=\hbar=1$; atomic units are used throughout the article). The potential energies $V_{1}(R)$ and $V_{2}(R)$ and the transition dipole moment were taken from [45, 46].

In the light-induced potential picture, after the absorption of one photon, the energy of the $V_{2}(R)$ excited electronic state is shifted downwards by $\hbar \omega_{L}$ - where $\omega_{L}$ is the laser frequency - creating a crossing between the diabatic ground and excited potential energy 
curves. After diagonalizing this diabatic potential energy matrix (eq. (2.1) so called lightinduced adiabatic states $V_{\text {lower }}$ and $V_{\text {upper }}$ are formed which can cross each other, creating a conical intersection whenever the conditions $\cos \theta=0,(\theta=\pi / 2)$ and $V_{1}(R)=V_{2}(R)-\hbar \omega_{L}$ are simultaneously met [3]. The position of this LICIs is determined by the laser frequency while the laser intensity controls the strength of the nonadiabatic coupling [3]. Increasing the frequency moves the CI to a smaller internuclear distance and to a lower energetic position while the opposite holds when decreasing the frequency.

\section{THE DETAILS OF THE CALCULATIONS}

Here a short description is given of the time-dependent (TD) electric fields of the pulses and of the wave packet propagation method used to solve the TD nuclear Scrödinger equation employing the Hamiltonian of eq. 2.1.

\section{A. The applied electric fields}

At time $t=0$ the system is ionized and the vibrational wave packet on the ground ionic state starts to oscillate on the $V_{1}=1 s \sigma_{g}$ potential curve. The initial nuclear wave packet is built up as the FC distribution of the ionic vibrational states and the molecule was initially in its rotational ground state $(\mathrm{J}=0)$. Then a laser pulse is applied after a time delay of $t_{\text {delay }}$. A linear polarized electric field is considered which is a product of a Gaussian envelope and a $\cos (\phi(t))$ functions

$$
E(t)=\epsilon_{0} f(t) \cos (\phi(t))
$$

Here $\epsilon_{0}$ is the maximum amplitude of the electric field and $f(t)=e^{-\frac{1}{2 \sigma^{2}}\left(t-t_{\text {delay }}\right)^{2}},(\sigma=$ $T_{p} / \sqrt{4 \log 2}$ ) is the Gaussian shape of the pulse. $T_{p}$ and $t_{\text {delay }}$ are the pulse duration (at FWHM) and the temporal maximum of the pulse, respectively. $\omega(t)=\frac{d}{d t} \phi(t)$ is the laser frequency and its time dependence is the control knob. The $\omega(t)=V_{2}(R)-V_{1}(R)$ function $(\hbar=1)$ controls the position of the nuclear coordinate $R$ at which the resonance condition for the appearance of the LICI is fulfilled. The initial nuclear wave packet is built up as the FC distribution of the ionic vibrational states and its field-free time evolution can be 
seen in Fig. 2A. The time period of the field-free oscillation is about $24 \mathrm{fs}$. As the wave packet is a superposition of several different vibrational eigenstates, it spreads during the time evolution relatively quickly, but exhibits recurrences. In order to force the LICI to move together with the field-free vibrational wave packet, we estimate the time-dependent frequency $\omega(t)$ from the motion of the field-free wave packet shown in Fig. 2A.

In our calculations the pulse duration was chosen to be $T_{p}=6 \mathrm{fs}$ in all examples studied, $t_{\text {delay }}$ was varied in the range between 0 to $100 \mathrm{fs}$, and the phase of the electric field at time $t$ was derived according to the following formula:

$$
\phi(t)=\int_{0}^{t} \omega\left(t^{\prime}\right) d t^{\prime} .
$$

In the present numerical study three kinds of chirped $\omega(t)$ functions were applied. They were obtained as explained in the following and are shown in Fig. 2B. The so called $\omega_{\text {global }}(t)$ function (red line on Fig. 2B) is obtained by following the global maximum of the density of the field-free wave packet $\left(\omega_{\text {global }}(t)=V_{2}\left(R_{\text {global }}(t)\right)-V_{1}\left(R_{\text {global }}(t)\right)\right)$ shown in Fig. 2A. This function is seen to exhibit abrupt strong oscillations at certain times. In order to get rid of these undesirable oscillations we applied a technique described below and obtained the so-called $\omega_{\text {local }}(t)$ function (black line in Fig. 2B). Instead of following the global maximum of the density of the nuclear wave packet, this function follows the maximum value of the local density of the wave packet $\left(\omega_{\text {local }}(t)=V_{2}\left(R_{\text {local }}(t)\right)-V_{1}\left(R_{\text {local }}(t)\right)\right)$. For deriving the $R_{\text {local }}(t)$ at time $t$, the maximum of the product of the density $|\psi(R, t)|^{2}$ and an exponential weight factor was considered rather than the maxima of the density themselves. This was performed by maximizing the following expression at each value of $t$ :

$$
|\psi(R, t)|^{2} e^{-\frac{1}{2 \sigma_{R}^{2}}\left(R-R_{\text {local }}(t-\Delta t)\right)^{2}} .
$$

Here $R_{\text {local }}(t-\Delta t)$ is the position of maximum in the preceding time step $(\Delta t=0.1 \mathrm{fs})$ and $\sigma_{R}$ has a value of 0.77 a.u. for $t<80$ fs and 0.28 a.u. for later times.

Finally, the third kind of chirped $\omega(t)$ function was simply obtained by following the average value $\langle R>$ of the variable $R$ as a function of time using as usual $<R(t)>=<$ $\psi(R, t)|R| \psi(R, t)>$. This chirped frequency function is named $\omega_{<R>}(t)$.

Since the pulses used in the present calculations are relatively short, we also compare the results with those of pulses of fixed frequencies, where for each time delay this constant 
frequency is chosen to correspond to $\omega_{\text {local }}(t)$ at that time delay. We will address the respective calculations as frequency varying transform limited (vTL) calculations. In many experiments a particular pulse is used for all time delays, and we have, therefore, also performed a reference transform limited (TL) calculation where the central frequency is the same $(\omega=0.23518$ a.u. $)$ at all time delays.

In the simulations the intensities $I=1 \times 10^{12} \frac{\mathrm{W}}{\mathrm{cm}^{2}}$ and $I=1 \times 10^{13} \frac{\mathrm{W}}{\mathrm{cm}^{2}}$ were applied. We note that the time integrals over the electric fields of the chirped pulses applied in the present work are small but not exactly zero[56].

\section{B. The wave packet propagation}

The dissociation dynamics in the LICI framework is described by solving the timedependent nuclear Schrödinger equation (TDSE) with the Hamiltonian $H$ described by eq. (2.1). For this the MCTDH (multi configuration time-dependent Hartree) method has been utilized [47 51]. For characterizing the vibrational degree of freedom we have used FFT-DVR (Fast Fourier Transformation-Discrete Variable Representation) with $N_{R}$ basis elements distributed within the range from $0.1 \mathrm{a} . \mathrm{u}$. to $80 \mathrm{a} . \mathrm{u}$. for the internuclear separation. The rotational degree of freedom was described by Legendre polynomials $\left\{P_{J}(\cos \theta)\right\}_{j=0,1,2, \cdots, N_{\theta}}$. These so called primitive basis sets $(\chi)$ were used to represent the single particle functions $(\phi)$, which in turn were used to represent the wave function:

$$
\begin{aligned}
\phi_{j_{q}}^{(q)}(q, t) & =\sum_{l=1}^{N_{q}} c_{j_{q} l}^{(q)}(t) \chi_{l}^{(q)}(q) \quad q=R, \theta \\
\psi(R, \theta, t) & =\sum_{j_{R}=1}^{n_{R}} \sum_{j_{\theta}=1}^{n_{\theta}} A_{j_{R}, j_{\theta}}(t) \phi_{j_{R}}^{(R)}(R, t) \phi_{j_{\theta}}^{(\theta)}(\theta, t) .
\end{aligned}
$$

The actual number of primitive basis functions in the numerical simulations were chosen to be $N_{R}=2048$ and $N_{\theta}=61$ for the vibrational and rotational degrees of freedom, respectively. On both diabatic surfaces and for both degrees of freedom a set of $n_{R}=n_{\theta}=4, \cdots, 20$ single particle functions were applied to form the nuclear wave packet of the system. (The actual value of $N_{\theta}$ and $n_{R}=n_{\theta}$ was chosen depending on the peak field intensity $I_{0}$.) Attention has been paid to the proper choice of basis so that convergence has been reached in each propagation. 
The TD nuclear wave packet is used to calculate the total dissociation probability $P_{\text {diss }}$ and the populations. The probability reads

$$
P_{\text {diss }}=\int_{0}^{\infty} d t\langle\psi(t)|W| \psi(t)\rangle .
$$

where $-i W$ is the complex absorbing potential (CAP) applied at the last $10 a . u$. of the vibrational $(R)$ grid.

To obtain the population of the individual vibrational levels we have used two steps of the simulation. The first one is initiated from the FC state averaged nuclear wave function and contains the interaction to the external electric field and the dissociation process. It is technically finished when the CAP eliminates the flying away portion of the wave packet $(t=350 \mathrm{fs})$. The second step of the simulation uses the final wave function of the first one as an initial wave function. From this second simulation we calculate the autocorrelation function as:

$$
C(t)=<\psi(R, \theta, 0) \mid \psi(R, \theta, T)>
$$

where $T$ is the time period of the second simulation. Next, we calculate the Fourier transform of this function to get the spectra of the bounded wave function [52 54]:

$$
\sigma(\omega)=\frac{1}{\pi} \int_{0}^{T} \Re\left[C(t) \cdot e^{i \omega t}\right] \cdot e^{-t / \tau} \cdot \cos ^{2} \frac{\pi t}{2 T} \cdot d t .
$$

The $\tau$ damping parameter was chosen to be $400 \mathrm{fs}$. Using a long enough second simulation $(T=350 \mathrm{fs})$ we can resolve the individual lines of the vibrational levels in the spectra. Finally, we integrate these individual peaks to get the population of the corresponding vibrational level:

$$
P_{\nu}=\int_{\left(E_{\nu-1}+E_{\nu}\right) / 2}^{\left(E_{\nu}+E_{\nu+1}\right) / 2} \sigma(\omega) d \omega
$$

\section{RESULTS AND DISCUSSION}

In our previous work linearly chirped laser pulses were used, which can easily be produced experimentally [55]. To be specific, the pulses preserved the spectrum of their transform limited (TL) equivalents and their time integral was exactly equal to zero. In this work, our attention is on constructing pulses in which the time dependence of the frequency is such 
that the light-induced conical intersection moves together with the field-free vibrational wave packet. This could be a first step towards developing a control protocol by conical intersection, and it is meaningful, because in case of short laser pulses the field-free wave packet does not change dramatically during the dynamical process. We are interested in finding out how much this can enhance the dissociation yield of the $D_{2}^{+}$ion and also studying how such a chirp influences the behavior of the photodissociation probabilities as a function of delay time and the populations on the different vibrational levels. Throughout the paper $T_{p}=6 f s$ pulse length is used.

\section{A. Dissociation probability}

\section{Chirped pulses}

Figure 3 (panel A) shows the total dissociation probability (Eq. (3.5)) as a function of time delay for the TL and for the chirped simulations employing a $1 \times 10^{12} \frac{\mathrm{W}}{\mathrm{cm}^{2}}$ intensity. The central frequency of the TL pulse is the same for all particular time delays and its value ( $\omega=0.23518$ a.u.) corresponds to the value of the second minimum of the $\omega_{<R>}(t)$ function in Fig. 2B. Three different chirped $\omega(t)$ functions $\left(\omega_{\text {global }}(t), \omega_{\text {local }}(t)\right.$ and $\left.\omega_{<R>}(t)\right)$ were applied in the simulations.

The most striking phenomenon is that each dissociation probability curve shows a periodic oscillatory behavior. One can easily understand it for the case of TL calculation, as in this situation the wave packet is periodically located at a distance where the resonance condition is satisfied, i.e. the LICI is positioned at the high density region of the wave packet. One might expect more balanced results over the time for the other curves, since in those cases the resonance condition is almost always fulfilled in such places, where the density of the wave packet possesses great value. Instead, we obtained curves which are more or less in sync with the TL one, possessing well defined maxima and minima and the ratios between these extreme values are significantly large. The latter is true both for the $\omega_{\text {global }}(t)$ and $\omega_{\text {local }}(t)$ curves, but perhaps it is more relevant for the case of $\omega_{\text {global }}(t)$. This is probably due to the finding that at larger nuclear distances the resonance condition induces dissociation more efficiently than at shorter distances. Indeed, starting, for instance, from $t_{\text {delay }}=35 \mathrm{fs}$, when the position of the actually selected local maximum of the nuclear 
density significantly differs from the position of the global time maximum, the $\omega_{\text {local }}(t)$ curve gives the higher dissociation yield. Comparing Figures 24 and $3 \mathrm{~A}$ we see that the large values of the dissociation rate correspond to large internuclear distances. That is, if the resonance condition is satisfied at larger internuclear distances a more efficient dissociation will result. Therefore, by determining the $\omega_{\text {local }}(t)$ function we have chosen that particular maximum value of the wave packet density from all the possible maximum values which belongs to a larger internuclear distance. It can be seen that the dissociation rates obtained by the $\omega_{\text {global }}(t)$ and $\omega_{\text {local }}(t)$ functions go well together up to $32-34 f s$ because in this case the positions of the global and the chosen local maximum density values coincide with each other. After $t_{\text {delay }}=34 \mathrm{fs}$, however, with a few exceptions, the $\omega_{\text {local }}(t)$ function always provides better results, i.e., higher dissociation probabilities. Although the first strong deviation between the $\omega_{\text {global }}(t)$ and $\omega_{\text {local }}(t)$ functions occurs at around $t_{\text {delay }}=39 \mathrm{fs}$, the impact of this deviation on the dissociation rate has already been felt at the end of the pulses centered at previous delay times. This manifests itself in the dissociation yield from $t_{\text {delay }}=34 \mathrm{fs}$ onwards.

Let us now discuss the results obtained with $\omega_{<R>}(t)$ frequency function which has been determined by following the time-dependent average of the internuclear distance. Although only marginally, the dissociation probability obtained by the $\omega_{<R>}(t)$ frequency function is the largest one up to three quarters of the first period of the oscillation of the field-free nuclear wave packet $\left(t_{\text {delay }}=18 f s\right)$. Accordingly, in this interval - when the wave packet is relatively well localized - it is more beneficial to adjust the $\omega(t)$ function to the timedependent average of the internuclear distance $<R>$ than to the maximum of the nuclear wave packet density. For $t_{\text {delay }}>18 \mathrm{fs}$ however, this dissociation yield (with $\omega_{<R>}(t)$ ) is basically always the lowest and even by far so at large time delays. The reason for this behavior can be found in Figure 2A. It is clearly seen that for $t_{\text {delay }}>18 f s$ the $\omega_{<R>}(t)$ function avoids the internuclear distances which correspond to the largest global or any local nuclear density values.

The dissociation probabilities for the pulses with $1 \times 10^{13} \frac{\mathrm{W}}{\mathrm{cm}^{2}}$ peak intensity are shown in Fig. 3B. The situation is roughly similar to that discussed above for the previous case of $1 \times 10^{12} \frac{\mathrm{W}}{\mathrm{cm}^{2}}$ peak intensity, but there are differences as well. It is eye-catching that while the intensity has increased by a factor of 10, the maximum yield of the dissociation increased only about 5 -fold. This implies that the dissociation probability has begun to saturate 
at this intensity. This is also related to the observation that while at the lower intensity $\omega_{\text {local }}(t)$ provides the highest dissociation yields between the $t_{\text {delay }}=35-50 \mathrm{fs}$, now at higher intensity, the yield curves obtained with $\omega_{\text {local }}(t)$ and $\omega_{\text {global }}(t)$ go well together up to $t_{\text {delay }}=40 \mathrm{fs}$. This saturation also affects several other features of the curves. For example, while at lower intensity in the $t_{\text {delay }}=40-50 \mathrm{fs}$ interval the $\omega_{\text {local }}(t)$ dissociation curve goes above the $\omega_{\text {global }}(t)$ one, the opposite is true at the higher intensity. This is in spite of the finding that the local nuclear density maxima are located at large internuclear distances and the dissociation process is more efficient here. Due to saturation, the large nuclear density present at small internuclear distances together with the 10-fold higher intensity all together produces more dissociation. Saturation is also responsible for finding that the differences between the dissociation yields obtained with various frequency function are slightly smaller than found at low intensity. All together, however, the use of $\omega_{\text {local }}(t)$ clearly provides the best choice for obtaining the largest dissociation yield.

In our previous studies [18, 19] the performance of TL and linearly chirped laser pulses in obtaining the dissociation probability has been investigated. There, the pulses used have fulfilled several conditions. We stress again that in the present work the time-dependence of the frequency functions of the pulses is designed in a completely different way following a different objective. As a consequence, the conditions put forward in [18, 19] do not hold anymore. Due to the different choice of the chirped pulses used in this work, a considerable growth of the total dissociation yield is obtained compared to the former results (see Fig. $3 \mathrm{~B}$ in [19]).

\section{Varying transform limited pulses}

In the varying transform limited (vTL) calculations, the frequency of the laser pulse was kept constant in every individual calculations at each time delay, corresponding to the $\omega$ value given by the $\omega_{\text {local }}(t)$ function at the respective time delay. The results for the dissociation rates obtained by the vTL and $\omega_{\text {local }}(t)$ pulses as well as by the TL reference pulse are displayed in Fig. 4

Inspecting the curves, we see that across the whole time interval the dissociation probabilities calculated by vTL pulses are smaller than those obtained by $\omega_{\text {local }}(t)$, except for the tiny $t_{\text {delay }}=8-12 \mathrm{fs}$ interval. In this time period the LICI corresponding to the 
constant frequencies of the vTL pulses is located at large internuclear distances during the pulse duration providing a more efficient dissociation, while in the case of the chirped pulse this applies only for the center of the pulse. However, because the nuclear wave packet is well localized in space during this time and its average momentum is close to 0 , there is no significant difference in the nuclear densities corresponding to the two different positions of the LICI. This results in a higher dissociation yield in this short time interval.

$\mathrm{Up}$ to $t_{\text {delay }}=18 \mathrm{fs}$ the TL and vTL pulses provide very similar results and this applies at many other time delays too. Such time periods are e.g. $t_{\text {delay }}=28-30 \mathrm{fs}$ and $t_{\text {delay }}=$ $50-55 \mathrm{fs}$, at which the dissociation yield shows a growing trend. This is surprising, because the vTL and TL pulses work on different frequencies. Mostly, the dissociation yield obtained by the TL pulses exhibits a smooth behavior. In particular beyond $t_{\text {delay }}=50 \mathrm{fs}$, the vTL results do not lie on a smooth curve. This is related to the fact that several islands of large density regions of the nuclear wave packet exist. Since the vTL dissociation yield is calculated by varying the time delay by $1 \mathrm{fs}$ steps, the frequency at two consecutive calculations can refer to two different islands and thus be rather different leading to the non-smooth behavior see in Fig. 4 .

As time proceeds, the peaks in the dissociation yield as a function of time delay become broader in the vTL calculations than in the reference TL curve. This implies that it is beneficial to use a varying frequency in order to increase the total dissociation probability. Although the pulse duration is rather short $\left(T_{p}=6 \mathrm{fs}\right)$, it is even more beneficial to use the $\omega_{\text {local }}(t)$ frequency function where the frequency changes during the pulse. As seen in Fig. 4. the gain is then substantial.

\section{B. Populations of the individual vibrational levels after the pulse is over}

We now examine the effect of photodissociation dynamics initiated by the $\omega_{\text {global }}(t)$ chirped laser pulses for the population of the different individual vibrational levels of the

residual $D_{2}^{+}$ion which has not undergone dissociation as the pulse terminated. Comparison has been made between the initial FC population of the $D_{2}^{+}$molecule and the population of the different vibrational levels after the dissociation has taken place. The results are collected in Table 1.

At the bottom of the table the actual energy positions of the LICI corresponding to 


\begin{tabular}{|c|c|c|c|c|c|c|c|c|}
\hline \multirow[b]{2}{*}{$\nu$} & \multirow[b]{2}{*}{$E_{\nu}$} & \multirow[b]{2}{*}{$P_{\nu}^{F C}$} & \multicolumn{2}{|c|}{$t_{\text {delay }}=8 \mathrm{fs}$} & \multicolumn{2}{|c|}{$t_{\text {delay }}=30 \mathrm{fs}$} & \multicolumn{2}{|c|}{$t_{\text {delay }}=59 \mathrm{fs}$} \\
\hline & & & $\Delta P_{\nu}$ & $\Delta P_{\nu} / P_{\nu}^{F C}$ & $\Delta P_{\nu}$ & $\Delta P_{\nu} / P_{\nu}^{F C}$ & $\Delta P_{\nu}$ & $\Delta P_{\nu} / P_{\nu}^{F C}$ \\
\hline 0 & -2.69 & 0.03655 & -0.00009 & $-0.23 \%$ & -0.00485 & $-13.27 \%$ & 0.00252 & $6.90 \%$ \\
\hline 1 & -2.49 & & 0489 & & -0.02095 & & 0.00170 & $1.77 \%$ \\
\hline 2 & -2.30 & 72 & 2038 & -14. & -0.04211 & & -0.03123 & $-21.73 \%$ \\
\hline 3 & -2.11 & 0.1 & 411 & -27. & -0.05510 & $-34.32 \%$ & -0.06932 & $-43.18 \%$ \\
\hline 4 & -1.94 & 0.14 & -0.06280 & $-41.94 \%$ & -0.05652 & $-37.74 \%$ & -0.07537 & $-50.33 \%$ \\
\hline 5 & -1.77 & 0.12374 & -0.06475 & $-52.32 \%$ & -0.05176 & $-41.83 \%$ & -0.05902 & $-47.69 \%$ \\
\hline 6 & -1.61 & 0.09385 & -0.05115 & $-54.50 \%$ & -0.04408 & $-46.97 \%$ & -0.04995 & $-53.23 \%$ \\
\hline 7 & -1.46 & 0.06693 & -0.03216 & & -0.03289 & & -0.04012 & $-59.94 \%$ \\
\hline 8 & -1.31 & 0.04 & -0.01569 & $-34.37 \%$ & -0.01633 & $-35.78 \%$ & -0.01018 & $-22.30 \%$ \\
\hline 9 & -1.17 & 0.03013 & -0.00512 & $-16.98 \%$ & -0.00154 & $-5.12 \%$ & 0.01627 & $54.01 \%$ \\
\hline 10 & -1.04 & 0.01 & 0.00066 & & 0.00350 & $18.00 \%$ & 0.0 & $19.10 \%$ \\
\hline 11 & -0.92 & 0.01 & 0.00274 & 22. & 0.00071 & $5.76 \%$ & -0.00420 & $-33.99 \%$ \\
\hline 12 & -0.80 & 0.00779 & 0.00309 & $39.71 \%$ & -0.00055 & $-7.12 \%$ & 0.00352 & $45.20 \%$ \\
\hline 13 & -0.70 & 0.00489 & 0.00274 & $56.00 \%$ & -0.00050 & $-10.31 \%$ & -0.00033 & $-6.85 \%$ \\
\hline 14 & -0.60 & 0.00 & & & -0.00027 & & -0.0 & $-26.24 \%$ \\
\hline 15 & -0.50 & 0.00 & 156 & & 0.00012 & & & $29.37 \%$ \\
\hline 16 & -0.42 & 0.00 & 0.00111 & $\%$ & -0.00017 & $-13.86 \%$ & 0.00045 & $36.61 \%$ \\
\hline 17 & -0.34 & 0.00 & 0077 & & 0.00003 & & 0.00019 & $24.25 \%$ \\
\hline 18 & -0.28 & 0.00 & 0.0 & 103. & 0.00002 & & 0.00005 & $10.12 \%$ \\
\hline 19 & -0.21 & 0.00033 & 0.00036 & $106.16 \%$ & -0.00009 & $-26.25 \%$ & -0.00007 & $-22.66 \%$ \\
\hline 20 & -0.16 & 0.00 & & & 0.00005 & & -0.00001 & $-4.16 \%$ \\
\hline 21 & -0.11 & & & & 0.00003 & & & $2370 \%$ \\
\hline 22 & -0.08 & 0.00010 & 0.00011 & $106.53 \%$ & -0.00003 & $-28.77 \%$ & -0.00002 & $-21.05 \%$ \\
\hline 23 & -0.05 & 0.00006 & 0.00007 & $104.77 \%$ & -0.00002 & $-33.46 \%$ & 0.00001 & $10.44 \%$ \\
\hline 24 & -0.02 & 0.00004 & 0.00004 & $100.79 \%$ & 0.00000 & $-7.41 \%$ & 0.00002 & $59.29 \%$ \\
\hline 25 & -0.01 & 0.00003 & 0.00003 & $97.74 \%$ & 0.00000 & $14.39 \%$ & 0.00000 & $13.71 \%$ \\
\hline \multicolumn{3}{|c|}{$\sum_{\nu} \Delta P_{\nu}:$} & \multirow{2}{*}{\multicolumn{2}{|c|}{-0.28483}} & \multirow{2}{*}{\multicolumn{2}{|c|}{-0.32329}} & \multicolumn{2}{|l|}{-0.31155} \\
\hline \multicolumn{3}{|c|}{$E_{C I}\left(t=t_{\text {delay }}\right)[\mathrm{eV}]$} & & & & & & \\
\hline
\end{tabular}

Table I: The changes of the populations of the individual vibrational levels during the light matter interaction for three different simulations. In all three cases the intensity of the field was $1 \times$ $10^{13} \mathrm{~W} / \mathrm{cm}^{2}$ and the frequency of the electric field was applied according to the $\omega_{\text {local }}(t)$ function. In the first three columns the vibrational levels $(\nu)$, the corresponding energies $\left(E_{\nu}\right)$ and the initial populations $\left(P_{\nu}^{F C}\right)$ of the vibrational eigenstates are displayed. The next three main columns each containing two sub-columns contain the absolute and relative changes of the populations for the chosen $t_{\text {delay }}=8,30$ and $59 \mathrm{fs}$. In the bottom of the table the last two rows contain the sum of the absolute changes (the dissociation probability) and the energetic position of the LICI at time $t=t_{\text {delay }}$. 
the relevant delay times are indicated. At $t_{\text {delay }}=8 \mathrm{fs}$ and $t_{\text {delay }}=59 \mathrm{fs}$ the LICI lies energetically between the $\nu=2$ and $\nu=3$ vibrational levels, while at $t_{\text {delay }}=30 \mathrm{fs}$ it lies very close to the $\nu=1$ level. It is striking that in the close vicinity of the LICI the population drastically decreases and the depletion is continuously held up to the $\nu=8$ vibrational level. This is the interval from where the ion can easily dissociate. Then, above the $\nu=8$ vibrational level a sudden change occurs resulting in a different behavior of the populations for the studied delay times. While for $t_{\text {delay }}=8 \mathrm{fs}$ the population is continuously increasing above the $\nu=9$ vibrational level, for the other two delay times an alternating increase or decrease of the populations occur. Because of the strong nonadiabatic effects present it is difficult to explain this interesting reshuffling of the populations. Probably, the presence of the nonadiabatic effects can, in addition to the direct dissociation process, induce vibrational excitations resulting in a newly mixed population distribution among the various vibrational levels. This process can be dominant above $\nu=8$ level because in this region practically no dissociation occurs. The populations of the vibrational levels should be amenable to measurements.

\section{CONCLUSIONS}

Applying the light-induced conical intersection framework, numerical simulations have been performed for studying the photodissociation dynamics of the $D_{2}^{+}$molecular ion in order to understand better the relation between the evolving nuclear vibrational wave packet and the frequency chirping of the laser pulse. We presented a strategy to directly follow the time evolution of the nuclear wave packet meanwhile forcing it to move together with the LICI as much as possible. Based on this idea three different types of chirped frequencies were designed. The effects of the laser pulses constructed by using these $\omega_{\text {global }}(t), \omega_{\text {local }}(t)$ and $\omega_{<R>}(t)$ functions have been explored in terms of the total dissociation rate as a function of the time delay between the ionization of $\mathrm{D}_{2}$ and the pulse, as well as in terms of the population of the different vibrational levels after the pulse has terminated. We have found that of all $\omega$ functions applied so far, the $\omega_{\text {local }}(t)$ provides the overall highest yield for the

dissociation rate, which is probably close to the largest possible outcome or dissociation limit for the given pulse length and intensity values. 
It is found that employing a frequency which is adapted to the time delay in question is very beneficial for the dissociation rate. Moreover, although the pulses are rather short $\left(T_{p}=6 f s\right)$, it is clearly demonstrated that varying the frequency during the pulse, i.e., using a chirped pulse, gives rise to a further large gain in the dissociation rate. The reshuffling of the populations of the individual vibrational levels of the residual pulse after the pulse has terminated has been found to depend markedly on the time delay employed. These populations contain much information on nonadiabatic effects due to the LICI and should be amenable to experiments.

In our work the motion of the LICI was adjusted to the time evolution of the nuclear field-free wave packet. As we applied only relatively short laser pulses $\left(T_{p}=6 f s\right)$ it seems meaningful as a first approximation to use the field-free wave packet because the pulses have not caused any dramatic effect on the structure of wave packet. The present results are very encouraging and call for including the effect of the pulse on the nuclear wave packet in determining a more refined chirp function useful in particular for longer pulses and also for high intensity pulses. This will include new studies using laser pulses in which the time dependence is designed to follow the dynamical evolution of the realistic wave packet. Namely, by means of the feedback-optimized frequency chirped laser pulses one could try to guide the time evolution of a molecular system together with the LICI towards a desired target state. In any case, the LICI opens the door to investigate light-induced nonadiabatic phenomena.

\section{Acknowledgments}

The authors acknowledge the financial support by the Deutsche Forschungsgemeinschaft (Project ID CE10/50-3). The ELI-ALPS project (GOP-1.1.1-12/B-2012-000, GINOP-2.3.615-2015-00001) is supported by the European Union and co-financed by the European Regional Development Fund. For this work, the supercomputing service of NIIF has been used. The authors thank H.-D. Meyer for very helpful discussions about the MCTDH calculations.

[1] G. A. Worth and L. S. Cederbaum, Annu. Rev. Phys. Chem., 2004, 55, 127. 
[2] M. Baer, Beyond Born Oppenheimer: Electronic Non-Adiabatic Coupling Terms and Conical Intersections 2006, Wiley, New York.

[3] N. Moiseyev, M. Sindelka and L. S. Cederbaum, J. Phys. B., 2008, 41, 221001.

[4] M. Sindelka, N. Moiseyev and L. S. Cederbaum, J. Phys. B., 2011, 44, 045603.

[5] G. J. Halász, Á. Vibók, M. Sindelka, N. Moiseyev and L. S. Cederbaum, J. Phys. B., 2011, 44, 175102 .

[6] G. J. Halász, M. Sindelka, N. Moiseyev, L. S. Cederbaum and Á. Vibók, J. Chem. Phys. A., 2012, 116, 2636.

[7] G. J. Halász, Á. Vibók, M. Sindelka, L. S. Cederbaum and N. Moiseyev, Chem. Phys., 2012, $399,146$.

[8] G. J. Halász, Á. Vibók, N. Moiseyev and L. S. Cederbaum, Phys. Rev. A., 2013, 88, 043413.

[9] A. Natan, M. R. Ware and P. H. Bucksbaum, Book of Ultrafast Phenomena XIX Springer Proceedings in Physics Volume 2015, 162, 122.

[10] G. J. Halász, Á. Vibók and L.S: Cederbaum, J. Phys. Chem Lett., 2015, 6, 348.

[11] L. S. Cederbaum, Y. C. Chiang, Ph. V. Demekhin and N. Moiseyev, Phys. Rev. Lett., 2011, 106, 123001.

[12] Ph. V. Demekhin and L. S. Cederbaum, J. Chem. Phys., 2013, 46, 164008.

[13] J. Kim, H. Tao, J. L. White, V. S. Petrovi, T. J. Martinez and P. H. Bucksbaum, J. Phys. Chem. A., 2012, 116, 2758.

[14] M. E. Corrales, J. González-Vázquez, G. Balerdi, I. R. Solá, R. de Nalda and L. Bañares, Nature Chem., 2014, 6,785.

[15] I. R. Solá, J. González-Vázquez, R. de Nalda and L. Bañares: Strong field laser control of photochemistry. Phys. Chem. Chem. Phys., 17, 13183 (2015).

[16] A. Stolow, Nature Chem., 2014, 6, 759.

[17] J. Kim, H. Tao, T. J. Martinez, P. H. Bucksbaum, J. Phys. B., 2015, 48, 164003.

[18] A. Csehi, G. J. Halász, L. S. Cederbaum and Á. Vibók, J. Chem. Phys., 2015, 143, 014305.

[19] A. Csehi, G. J. Halász, L. S. Cederbaum and Á. Vibók, J. Chem. Phys., 2016, 144, 074309.

[20] J. Cao, C. J. Bardeen and K. R. Wilson, Phys. Rev. Lett., 1998, 80, 1406.

[21] J. Cao, C. J. Bardeen and K. R. Wilson, J. Chem. Phys., 2000, 113, 1898.

[22] V. S. Malinovsky and J. L. Krause, Eur. Phys. J. D., 2001, 14, 147.

[23] A. Datta, S. S. Bhattacharyya and B. Kim, Phys. Rev. A., 2002, 65, 043404. 
[24] E. Heesel, B. M. Garraway and J. P. Marangos, J. Chem. Phys., 2006, 124, 024320.

[25] A. Kirrander, H. H. Fielding and Ch. Jungen, J. Chem. Phys., 2010, 132, 024313.

[26] A. Kirrander, Ch. Jungen and H. H. Fielding, J. Phys. B., 2008, 41, 074022.

[27] A. Kirrander and H. H. Fielding, J. Phys. B., 2007, 40, 897.

[28] G. Katz, M. A. Ratner and R. Kosloff, New J. Phys. 2010, 12, 015003.

[29] J. Vala and R. Kosloff, Optics Express, 2001, 8, 238.

[30] L. Rybak, Z. Amitay, S. Amaran, R. Kosloff, M. Tomza, R. Moszynski and C. P. Koch, Faraday Discuss. Chem. Soc. 2011, 153, 383.

[31] L. Rybak, S. Amaran, L. Levin, M. Tomza, R. Moszynski, Z. R. Kosloff, C. P. Koch and Z. Amitay, Phys. Rev. Lett., 2011, 107, 273001.

[32] L. Levin, W. Skomorowski, L. Rybak, R. Kosloff, C. P. Koch and Z. Amitay, Phys. Rev. Lett., 2015, 114, 233003.

[33] V. S. Prabhudesai, U. Lev, A. Natan, B. D. Bruner, A. Diner, O. Heber, D. Strasser, D. Schwalm, I. Ben-Itzhak, J. J. Hua, B. D. Esry, Y. Silberberg, D. Zajfman, Phys. Rev. A. 2010, 81, 023401.

[34] A. Natan, U. Lev, V. S. Prabhudesai, B. D. Bruner, D. Strasser, D. Schwalm, I. Ben-Itzhak, O. Heber, D. Zajfman and Y. Silberberg, Phys. Rev. A 2012, 86, 043418.

[35] V. S. Prabhudesai, A. Natan, B. D. Bruner, Y. Silberberg, U. Lev, O. Heber, D. Strasser, D. Schwalm, D. Zajfman, I. Ben-Itzhak, J. Kor. Phys. Soc. 2011, 59, 2890.

[36] B. Y. Chang, S. Shin, J. Santamaria and I. R. Sola, J. Phys. Chem. A 2012, 116, 2691.

[37] S. Askeland and M. Førre, Phys. Rev. A 2013, 88, 043411.

[38] C. P. Zhang, X. Y. Miao, Spect. Lett. 2014, 47, 267.

[39] U. Lev, L. Graham, C. B. Madsen, I. Ben-Itzhak, B. D. Bruner. B. D. Esry, H. Frosting, O. Heber, A. Natan and V. S. Prabhudesai, J. Phys. B 2015, 48, 201001.

[40] D. V. Novitsky, Optic Comm. 2016, 358, 202.

[41] C. C. Shu and N. E. Henriksen, J. Chem. Phys., 2011, 134, 164308.

[42] C. C. Shu and N. E. Henriksen, J. Chem. Phys., 2012, 136, 044303.

[43] A. K. Tiwari, D. Dey and N. E. Henriksen, Phys. Rev. A 2014, 89, 023417.

[44] S. I. Chu, J. Chem. Phys. 1981, 75, 2215.

[45] S. I. Chu, C. Laughlin, and K. Datta, Chem. Phys. Lett. 1983, 98, 476.

[46] F. V. Bunkin and I. I. Tugov, Phys. Rev. A 1973, 8, 601. 
[47] H. D. Meyer, U. Manthe and L. S. Cederbaum, Chem. Phys. Lett. 1990, 165, 73.

[48] U. Manthe, H. D. Meyer and L. S. Cederbaum, J. Chem. Phys. 1992, 97, 3199.

[49] M. H. Beck, A. Jäckle, G. A. Worth and H. D. Meyer, Phys. Rep. 2000, 324, 1.

[50] G. A. Worth et al. The MCTDH package, version 8.2; University of Heidelberg: Heidelberg, Germany, 2000. H. D. Meyer et al. The MCTDH package, versions 8.3 and 8.4; University of Heidelberg, Germany, 2002 and 2007. http://mctdh.uni-hd.de/

[51] Multidimensional Quantum Dynamics: MCTDH Theory and Applications, edited by H. D. Meyer, F. Gatti, G. A. Worth, (Wiley-VCH: Weinheim, 2009).

[52] R. Schinke, Photodissociation Dynamics, Cambridge University Press, Cambridge, 1993.

[53] G. G. Balint-Kurti, R. N. Dixon and C. C. Marston, J. Chem. Soc., Faraday Trans. 1990, 86, 1741.

[54] O. Vendrell, F. Gatti and H.-D. Meyer, J. Chem. Phys. 2007, 127, 184303.

[55] D. B. Milosevic, G. G. Paulus, D. Bauer, and W. Becker, J. Phys. B 2006, 39, R203.

[56] For TL cases the ratio of the integrals relative to the Gaussian envelope are below $10^{-8}$, while for the other frequency functions $\left(\omega_{\text {local }}(t)\right.$ and $\left.\omega_{\text {global }}(t)\right)$ are below $1.5 \%$. 


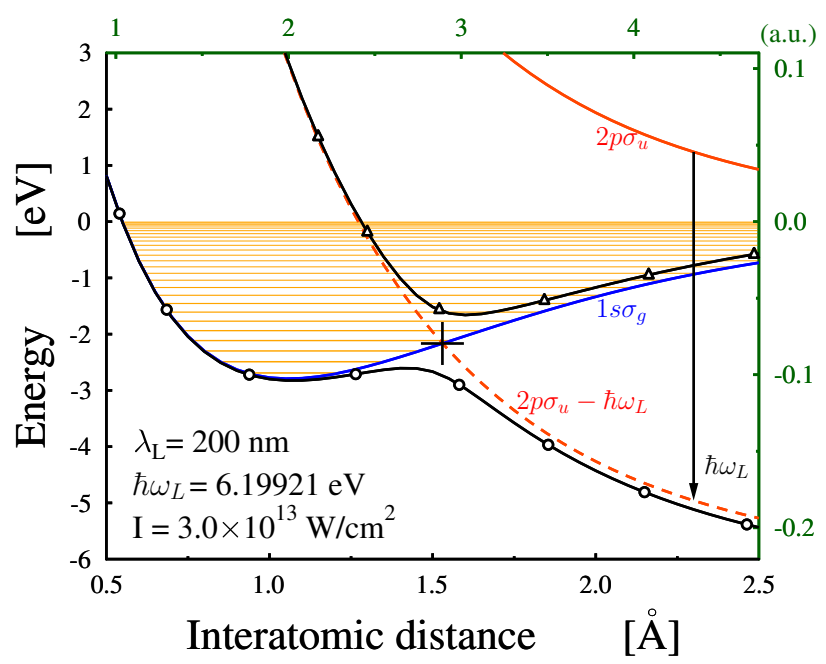

Figure 1: Potential energy curves of the $\mathrm{D}_{2}^{+}$ion. Diabatic energies of the ground $1 s \sigma_{g}$ and the first excited $2 p \sigma_{u}$ states are displayed with solid blue and red lines, respectively. The field dressed excited state $\left(2 p \sigma_{u}-\omega_{L}\right)$ is presented with dashed red line. The field dressed excited state $\left(2 p \sigma_{u}-\omega_{L}\right)$ forms a light-induced conical intersection (LICIs) with the ground state. A cut through the adiabatic surfaces at $\theta=0$ (parallel to the field) is also shown for a particular frequency $\left(\omega_{L}=6.19921 \mathrm{eV}\right)$ and field intensity $\left(3 \times 10^{13} \frac{\mathrm{W}}{\mathrm{cm}^{2}}\right)$ depicted by solid black lines marked with circles $\left(V_{l}\right)$ and triangles $\left(V_{u}\right)$. Alternative $\mathrm{x}-\mathrm{y}$ axises (top and right hand side) with a.u.'s are provided. The horizontal orange lines denote the field-free vibrational levels of the $1 s \sigma_{g}$ state. We denote with a cross the position of the LICI $\left(R_{L I C I}=1.53 \AA=2.891 a . u\right.$. and $\left.E_{L I C I}=-2.16611 \mathrm{eV}\right)$. 

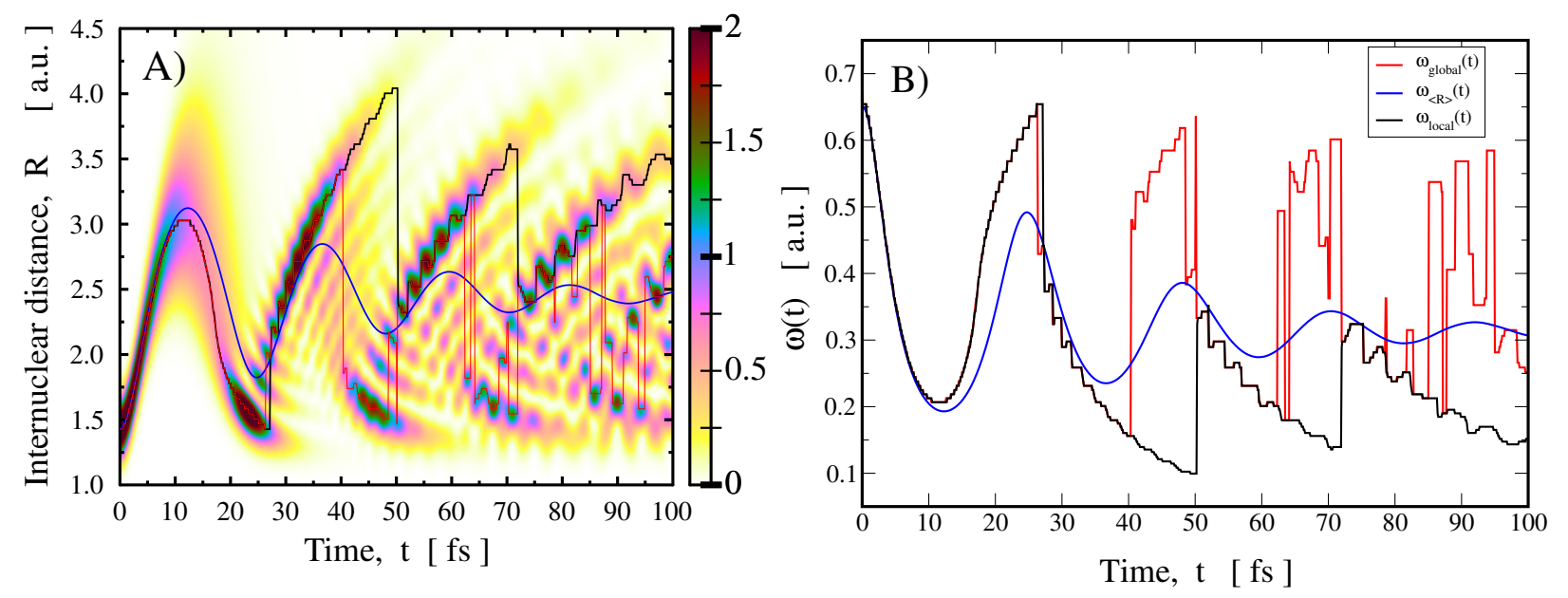

Figure 2: The field-free vibration of the $D_{2}^{+}$system as a function of time $t$ after ionization of the neutral at time $t=0$ and the deduced chirped pulse frequency. In Panel (A) the square of the vibrational wave packet is represented by color code: the darker the color the larger the amplitude of the wave packet. Additionally, three curves are shown which are used to determine the chirped frequency of the pulses employed in this investigation: The time-dependent average of the internuclear distance (blue curve), a curve following the global (red curve) and one following the local (black curve) maximum positions of the density of the field-free wave packet. For more details see the main text. Panel (B) shows the resulting three different forms of the $\omega(t)$ frequency function (for definition see in Section IIIA.) of the laser pulse used in the calculations of the nuclear dynamics. The respective frequency functions are denoted by $\omega_{<R>}(t), \omega_{\text {global }}(t)$ and $\omega_{\text {local }}(t)$. 

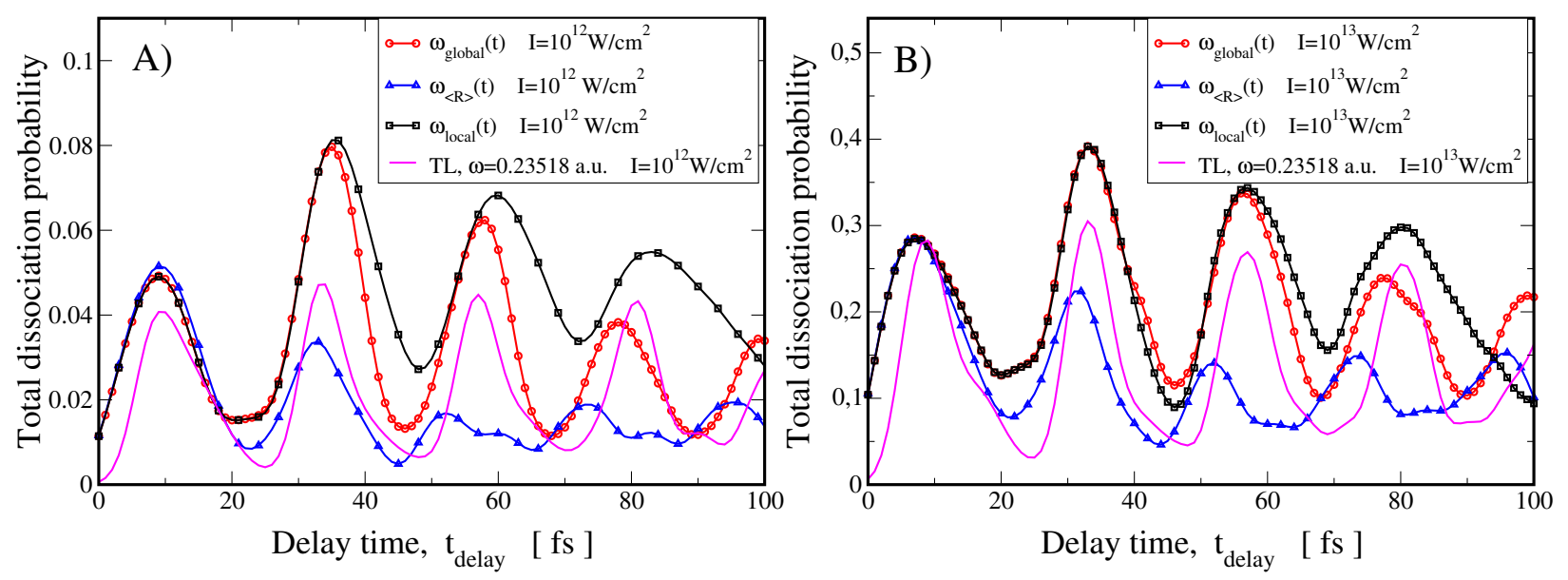

Figure 3: Dissociation probabilities of $\mathrm{D}_{2}^{+}$as a function of the delay time $\left(t_{\text {delay }}\right)$ of the pulse. In panel (A) the black (marked with square), the red (marked with circle) and the blue curves (marked with triangle) denote the dissociation probabilities corresponding to the $\omega_{\text {local }}(t), \omega_{\text {global }}(t)$ and $\omega_{<R>}(t)$ frequency functions, respectively, at $1 \times 10^{12} \frac{\mathrm{W}}{\mathrm{cm}^{2}}$ intensity. The fourth curve (magenta solid) shows the reference transform limited (TL) result calculated with $\omega=0.23518$ a.u. Panel (B) shows the same curves as in panel $(\mathrm{A})$, but for $1 \times 10^{13} \frac{\mathrm{W}}{\mathrm{cm}^{2}}$ intensity. In all calculations the pulse length is $T_{p}=6 \mathrm{fs}$.

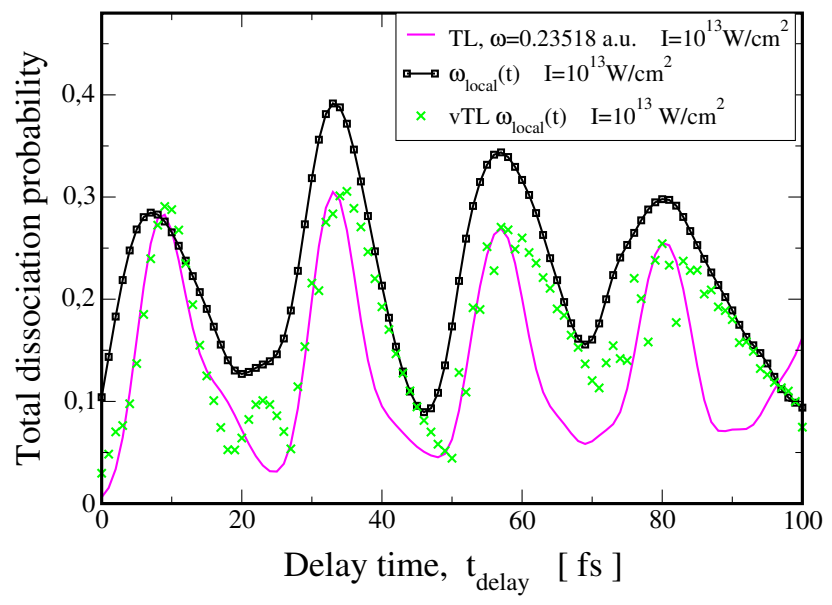

Figure 4: Dissociation probabilities of $\mathrm{D}_{2}^{+}$as a function of the delay time $\left(t_{\text {delay }}\right)$ of the pulse. The black (marked with square), the green crosses and the magenta (solid) curves show the dissociation probabilities corresponding to the $\omega_{\text {local }}(t)$, vTL $\omega_{\text {local }}(t)$ and the TL $\omega=0.23518$ a.u. frequencies, respectively. In the calculations the pulse length is $T_{p}=6 \mathrm{fs}$ and the intensity is $1 \times 10^{13} \frac{\mathrm{W}}{\mathrm{cm}^{2}}$. 